\title{
PALINOLOGía DE LOS GÉNEROS MODIOLA, MODIOLASTRUM Y TROPIDOCOCCUS (MALVACEAE)
}

\author{
GRACIELA ANA CUADRADO
}

Summary: Cuadrado, G.A. 2003. Palynology of the genera Modiola, Modiolastrum and Tropidococcus (Malvaceae). Bonplandia 12(1-4): 67-82. ISSN: 0524-0476.

Pollen grains of seven species of three genera of Malvaceae are studied and described under optical and scanning microscopy: Modiola: M. caroliniana (L.) G. Don, Modiolastrum: M. australe Krapov., M. gilliesii (Steud.) Krapov., M. lateritium (Hook.) Krapov., M. malviflorum (Griseb.) K. Schum., M. palustre (Ekman) Krapov. and Tropidococcus: T. pinnatipartitus (A. St.-Hil. \& Naudin) Krapov. They show clear differences at generic level and it is also possible to distinguish species, which in some cases are based on less important phylogenetic pollen characters. Pollen grains are sub oblate, oblate spheroidal and spheroidal; medium to big sized. Surfaces do not show great variability; the seven species are echinate, with nanoverrucae or spinulae between spines. The apertures show variations in number (three to twelve) and distribution pattern: zonoaperturate, spiralloid arrangement and dizonoaperturate. The distinctive pollen of Tropidococcus pinnatipartitus (large sized, spheroidal, dizonoaperturate, number of apertures: twelve), supports its segregation from the genus Modiolastrum. A key to determine these seven species is given. The basic chromosomes number of the three genera were compared with the apertures number, showing that those with lower basic chromosomes number, have fewer apertures than those with high ones. Five pollen grains types are recognized based on the number and distribution pattern of apertures: 1) Type tricolporate: Modiolastrum malvifolium and $M$. palustre . 2) Type pentazonocolp(oid)orate: Modiolastrum australe y M.gilliesii. 3) Type pentacolp(oid)orate spiralloid arragement: $M$. lateritium. 4) Type hexacolp(oid)orado spiralloid arragement: Modiola caroliniana y 5) Typedizonocolp(oid)orate, arranged in series of six apertures at each side of the equator: Tropidococcus pinnatipartitus.

Key words: Pollen, morphology, taxonomy.

Resumen: Cuadrado, G.A. 2003. Palinología de los géneros Modiola, Modiolastrum and Tropidococcus (Malvaceae). Bonplandia 12(1-4): 67-82. ISSN: 0524-0476.

Fueron estudiados y descriptos los granos de polen de las especies de tres géneros de Malvaceae, Modiola: M. caroliniana (L.) G. Don, Modiolastrum: M. australe Krapov., M. gilliesii (Steud.) Krapov., M. lateritium (Hook.) Krapov., M. malviflorum (Griseb.) K. Schum., M. palustre (Ekman) Krapov. y Tropidococcus: T. pinnatipartitus (A. St.- Hil. \& Naudin) Krapov. A nivel genérico se identifican con facilidad e incluso es posible hacerlo a nivel específico sólo que en algunos casos, esto se consigue con caracteres de menor importancia filogenética. Los granos de polen son suboblatos, oblato esferoidales y esferoidales, de medianos a grandes. La ornamentación no muestra gran variabilidad y las siete especies son equinadas con nanoverrugas o espínulas entre ellas. Las aperturas son variadas en número (de tres a doce)

Cátedra de Palinología- Facultad de Cs. Exactas y Naturales y Agrimensura- UNNE. Centro de Ecología Aplicada (CECOAL)-CONICET.Ruta 5 km 2,5-C.C.291-(3400) Corrientes- Argentina. Tel/Fax 54-(03783)454417. E-mail: hcaplan@arnet.com.ar 
BONPLANDIA 12(1-4). 2003

\begin{abstract}
y posición zonocolp(oid)oradas, espiraloide y dizonoaperturadas. Las características diferentes del polen de Tropidococcus pinnatipartitus (grandes, esferoidales, dizonoaperturados, número de aperturas doce), sustentan su separación del género Modiolastrum. Se presenta una clave para identificar las especies. El número básico de cromosomas de los tres géneros fue comparado con el número de aperturas de los granos de polen, resultando tener menos, los que tenían número básico de cromosomas más bajo, que los que lo tenían más alto. Se reconocieron cinco tipos de granos de polen teniendo en cuenta el número y disposición de las aperturas: 1) Tipo trizonocolporado: Modiolastrum malvifolium y $M$. palustre. 2) Tipo pentazonocolp(oid)orado: Modiolastrum australe у $M$. gilliesii. 3) Tipo pentacolp(oid)orado en disposición espiraloide: M. latehtium. 4) Tipo hexacolp(oid)orado en disposición espiraloide: Modiola caroliniana y 5) Tipo dizonocolp(oid)orado, ordenado en dos series de seis aperturas a cada lado del ecuador: Tropidococcus pinnatipartitus.
\end{abstract}

Palabras clave: Polen, morfología, taxonomía.

\section{Introducción}

Esta contribución tiene por objeto complementar datos exomorfológicos y comparar el número básico de cromosomas en Modiola Moench, Modiolastrum K. Schum. y Tropidococcus Krapov. con el número de aperturas de los granos de polen, con la finalidad de comprobar si en este caso se cumple la idea de que, en general, el mayor número de aperturas indica un mayor grado de evolución (Erdtman 1963, Christensen 1986).

Krapovickas (2003) separa Modiolastrum pinnatipartitum (A. St.-Hil. \&Naudin) Krapov. del género Modiolastrum, basándose en la presencia de una quilla en el mericarpio y el número básico de cromosomas, $x=12$. Crea así el género monotípico Tropidococcus.

Se estudia el polen de la única especie del género Modiola: M. caroliniana (L.) G. Don., de las 5 especies del género Modiolastrum: $M$. australe Krapov., M. gilliesii (Steud.) Krapov., M. lateritium (Hook.) Krapov., M. malvifolium (Griseb.) K. Schum. y M. palustre (Ekman) Krapov., y de la única especie del género Tropidococcus. T. pinnatipartitus (A. St.-Hil. \& Naudin) Krapov. Los tres géneros pertenecen a la tribu Malvae.

Los trabajos de polen de Malváceas en nuestro país son escasos. Referente a los géneros Modiola y Modiolastrum no se hallaron trabajos de autores argentinos, pero en el contexto internacional existen trabajos previos.

Hashmi (1970) describe el polen de 19 géneros y 74 especies de Malvaceae de Texas, entre las que figura Modiola caroliniana.
Heusser (1971) en su obra "Pollen and Spores of Chile" incluye algunas especies de Malváceas entre las que se encuentra Modiola caroliniana. Ambos autores sólo utilizan microscopio óptico para sus descripciones.

Christensen (1986) estudió 120 especies pertenecientes a 45 géneros de Malvaceae procedentes de diferentes partes del mundo: Estados Unidos, México, Ecuador, Panamá, Venezuela, Brasil, Argentina, Sudáfrica, Australia, Nueva Zelandia, Tailandia, España, Afganistán, Irán, con microscopio óptico y algunos casos con microscopio electrónico. Uno de los objetivos de su trabajo fue describir el polen de esta familia y discutir aspectos evolutivos y filogenéticos. La autora describe el polen del género Modiola con su única especie $M$. caroliniana, examina el polen de dos especies de Modiolastrum: M. malvifolium $y$ M. gilliesii y realizó una sola descripción para ambas, para lo cual utilizó material procedente de Argentina. Sólo $M$. gilliesii fue observado con microscopio electrónico.

\section{Material y Métodos}

Los ejemplares utilizados en este estudio provienen del Herbario del Instituto de Botánica del Nordeste (CTES), y fueron identificados por el Ing. Antonio Krapovickas.

Se analizaron 2-4 ejemplares, según los casos, de distintas localidades. El material utilizado fue procesado con el método de acetólisis de Erdtman (1960) y montado en glicerina-gelatina. Los preparados obtenidos 
están depositados en la Palinoteca de la Universidad Nacional del Nordeste, PAL-CTES. Para la observación con el microscopio electrónico de barrido (MEB) se utilizaron granos de polen previamente acetolizados y montados en una platina metálica y bañados con oro.

Las observaciones y descripciones se realizaron con un microscopio óptico (MO) Olympus $\mathrm{BX} 40$ y las fotografías ópticas se obtuvieron con una cámara fotográfica Olympus SC 35. De cada muestra se midieron en un mínimo de 20 granos: longitud del eje polar (P), diámetro ecuatorial (E), diámetro de poros y espesor del engrosamiento, largo de colpoides, distancia entre las aperturas, altura de las espinas, distancia entre las mismas, espesor de la exina en la zona interespinal e infraespinal, diferencia en los espesores de sexina y nexina. La observación e interpretación de la escultura y de las aperturas se complementó con las fotografías tomadas con microscopio electrónico de barrido (MEB) Jeol JSM-580 OLV de la Secretaría General de Ciencia y Técnica de la Universidad Nacional del Nordeste.

La terminología utilizada es la de Erdtman (1966) y Punt et al. (1994).

\section{Material estudiado}

Modiola caroliniana (L.) G. Don: ARGENTINA. Corrientes: Mercedes-Paso de los Libres, $\mathrm{A}^{\circ}$ Miriñay, Schinini \& al. 18642 (CTES), PAL-CTES 6552; Empedrado, Estancia La Yela, Pedersen 12974 (CTES), PAL-CTES 6553.

Modiolastrum australe Krapov.: ARGENTINA. Buenos Aires: Bahía Blanca, Villamil 5628 (CTES), PAL-CTES 6554; Villamil 5160 (CTES), PALCTES 6555.

Modiolastrum gilliesii (Steud.) Krapov.: ARGENTINA. Entre Ríos: Gualeguay -Victoria, Troncoso \& Bacigalupo 2931 (CTES), PAL-CTES 6556; Paraná, Keller 12943 (CTES) PAL-CTES 6557. Corrientes: Goya, Krapovickas \& Cristóbal 22728 (CTES), PAL-CTES 6558.

Modiolastrum lateritium (Hook.) Krapov.: ARGENTINA. Misiones: San Pedro, Morrone \& al. 1508 (CTES), PAL-CTES 6560. Corrientes: San Miguel, Pedersen 12943 (CTES), PAL-CTES 6561 ; Santo Tomé, O. Ahumada \& Schinini 4087 (CTES), PALCTES 6559.
Modiolastrum malvifolium (Griseb.) K. Schum.: ARGENTINA. Chaco: General Güemes, Krapovickas \& al. 46839 (CTES), PAL-CTES 6562. Misiones: Santa Ana, Vanni \& al. 3900 (CTES), PAL-CTES 6569. Corrientes: Curuzú Cuatiá, Pedersen 15633 (CTES), PAL- CTES 6563; Curuzú Cuatiá, Schinini 11667 (CTES), PAL-CTES 6564.

Modiolastrum palustre (Ekman) Krapov.: BRASIL. Paraná: Contenda, Hatschbach 15231 (CTES) PAL-CTES 6567; Paraná: Curitiba, Río Atuba, Hastchbach 32784 (CTES), PAL-CTES 6568.

Tropidococcus pinnatipartitus (A. St.-Hil. \& Naudin) Krapov. BRASIL. Rio Grande do Sul, Cacapava do Sul, Pedersen 11472 (CTES), PALCTES 6565. Krapovickas \& Cristóbal 34209 (CTES), PAL-CTES 6566.

\section{Resultados}

\section{Modiola caroliniana (L.) G. Don}

Figs. 1 A y 2 A-F

Granos medianos, oblato esferoidales. Medidas: $\mathrm{P}=30(43,3) 48 \mu \mathrm{m} ; \mathrm{E}=32,5(44,3) 50$ $\mu \mathrm{m} . \mathrm{P} / \mathrm{E}=0.93$.

Hexacolp(oid)orados, aperturas dispuestas en disposición espiraloide. Ora circulares de 5 $\mu \mathrm{m}$ de diámetro y engrosamiento anular de 3 $\mu \mathrm{m}$; los colpoides miden $10 \mu \mathrm{m}$ de largo y se visualizan dificultosamente con el MO. La exina tiene un espesor de $3 \mu \mathrm{m}$ bajo las espinas, sexina $2 \mu \mathrm{m}$ y nexina $1 \mu \mathrm{m}$; en $;$ a zona interespinal la exina mide $2 \mu \mathrm{m}$, sexina $1 \mu \mathrm{m}$ y nexina $1 \mu \mathrm{m}$. La ornamentación está constituida por espinas de $3 \mu \mathrm{m}$ de altura y $2 \mu \mathrm{m}$ de base, con una distancia aproximada entre las mismas de $7 \mu \mathrm{m}$, alternando con elementos positivos, regularmente dispuestos.

MEB: Permite observar los colpoides con claridad. Alternando con las espinas, se observan nanoverrugas del orden de $1,5 \mu \mathrm{m}$ de altura, las que están distribuidas laxa e irregularmente y perforaciones que sólo se ven con altos aumentos (5000x) y tienen diferente tamaño y forma (fóveas y fósulas).

Distribución: Argentina (Chaco, Formosa, 

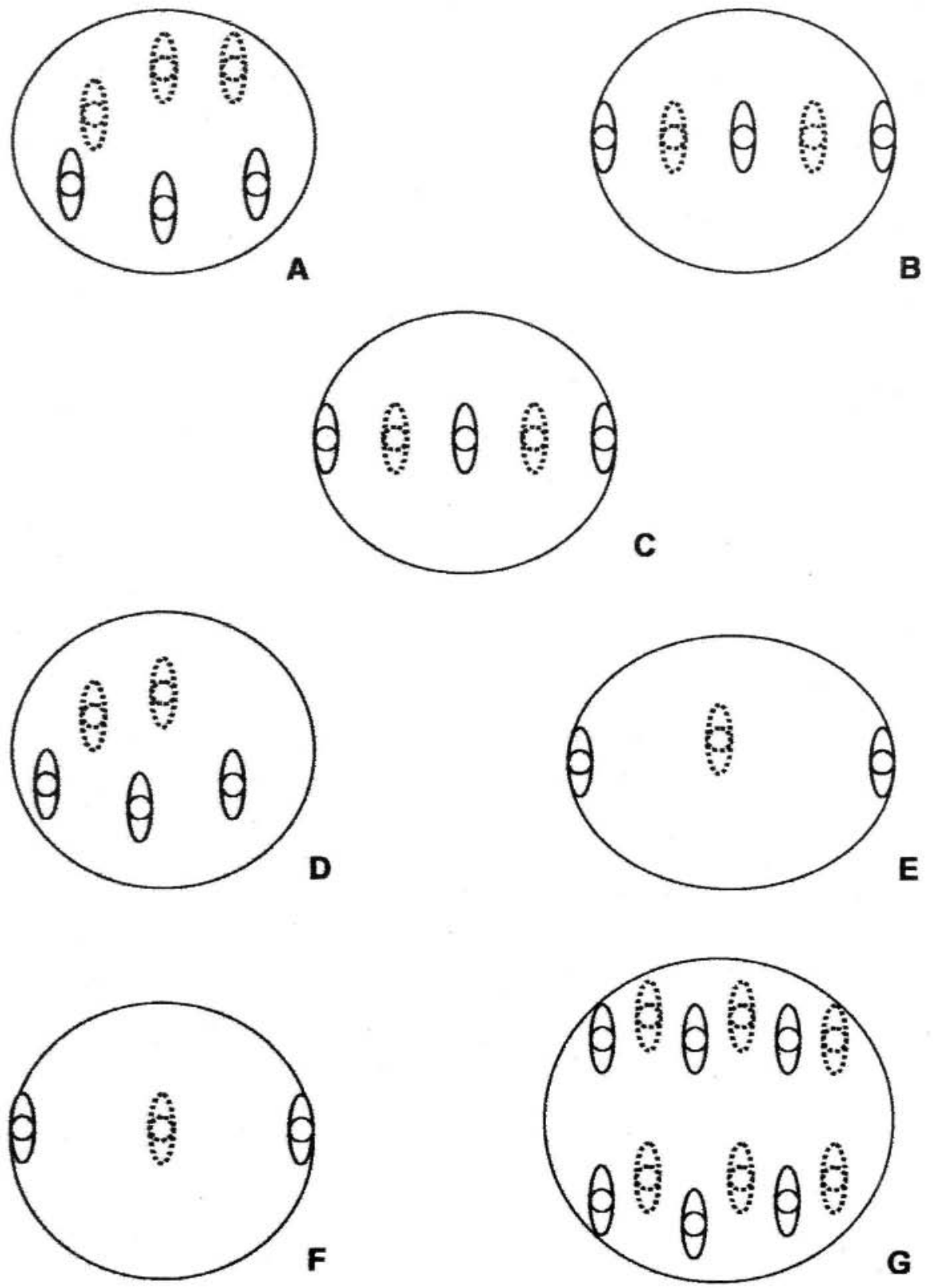

Fig.1. Esquemas representando la ubicación de las aperturas. A: Modiola caroliniana. B: Modiolastrum australe. C: Modiolastrum gilliesii. D: Modiolastrum lateritium. E: Modiolastrum malvifolium. F: Modiolastrum palustre. G: Tropidococcus pinnatipartitus. La escala equivale a $15 \mathrm{~mm}$ en A y B, a $6,5 \mathrm{~mm}$ en $\mathrm{C}$ y a $30 \mathrm{~mm}$ en D, E, F y G. 

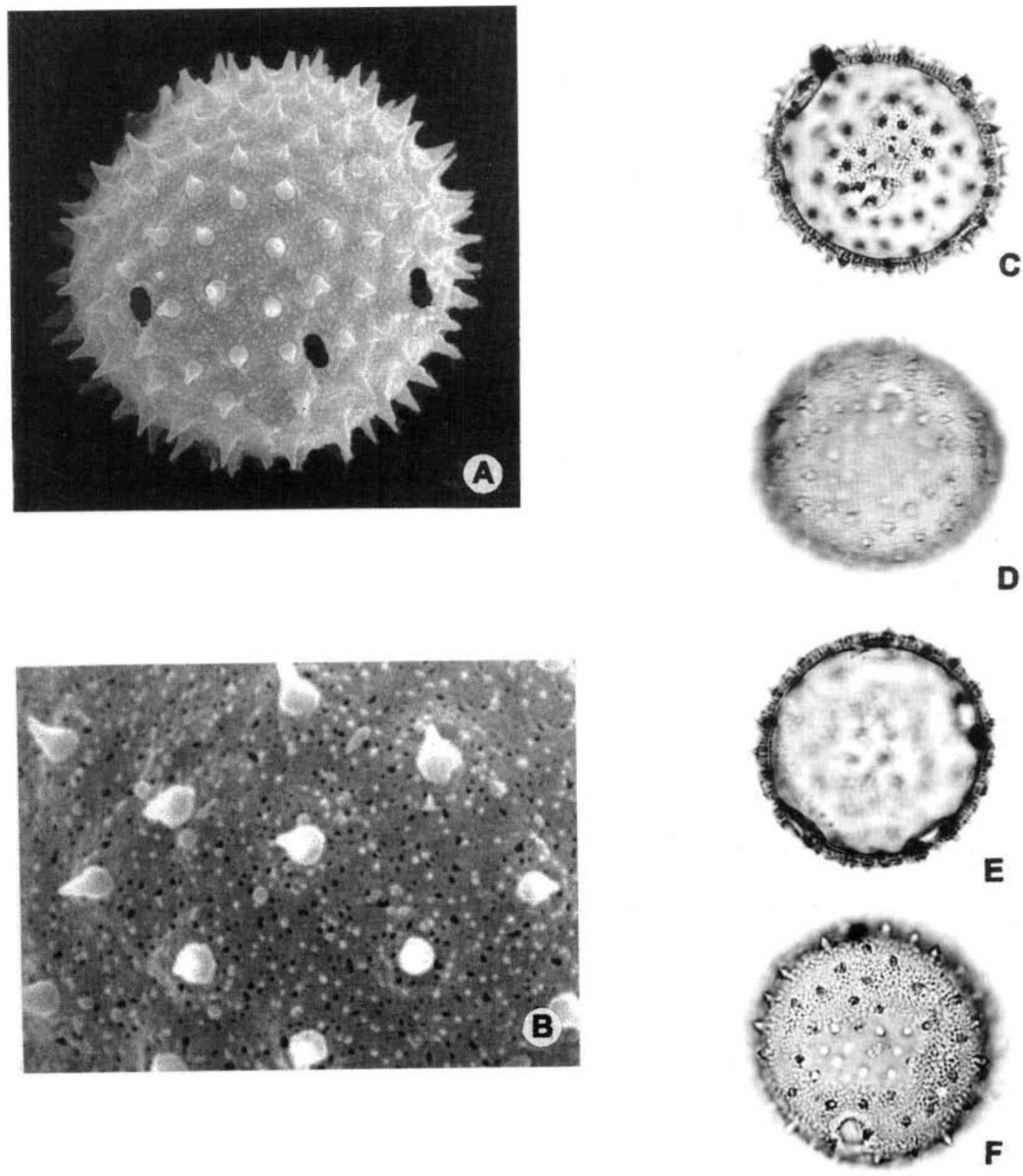

Fig. 2. Modiola caroliniana. MEB: A: vista ecuatorial, mostrando tres aperturas. B: detalle de escultura (5000x) (Pedersen 12974). MO: C: vista ecuatorial, foco inferior. D: vista ecuatorial, foco superior. E: vista polar, corte óptico. F: vista superficial (Schinini el al. 18642). La escala equivale a $15 \mathrm{~mm}$ en A y B, a $6,5 \mathrm{~mm}$ en $\mathrm{C}$ y a $30 \mathrm{~mm}$ en D, E y F. 
BONPLANDIA 12(1-4). 2003

Misiones, Corrientes, Mendoza, San Luis y Buenos Aires).

Modiolastrum australe Krapov.

Figs. 1 B y 3 A-F

Granos grandes, oblato esferoidales. Medidas: $\mathrm{P}=56(63) 70 \mu \mathrm{m} ; \mathrm{E}=58$ (70) $80 \mu \mathrm{m} . \quad V I$ $\mathrm{E}=0,90$

Zonocolp(oid)orados. Número de aperturas 5. Ora circulares de $7 \mu \mathrm{m}$ de diámetro y un engrosamiento muy angosto de $1,4 \mu \mathrm{m}$; los colpoides miden $16 \mu \mathrm{m}$ de largo y se visualizan dificultosamente con el MO. La exina tiene un espesor de $4 \mu \mathrm{m}$ bajo las espinas, sexina $2,5 \mu \mathrm{m}$ y nexina $1,5 \mu \mathrm{m}$; en la zona interespinal la exina mide $3 \mu \mathrm{m}$, sexina 1,5 $\mu \mathrm{m}$ y nexina $1,5 \mu \mathrm{m}$. La ornamentación está constituida por espinas de $6 \mu \mathrm{m}$ de altura, con una distancia aproximada entre las mismas de $5 \mu \mathrm{m}$, alternando con elementos positivos, regularmente dispuestos.

MEB: Permite observar que cada mamelón muestra en su base una disposición anular de nanoverrugas y escasas perforaciones. Entre las espinas, también alternan nanoverrugas.

Distribución: Argentina (Buenos Aires).

\section{Modiolastrum gilliesii (Steud.) Krapov.}

Figs. 1 C y 4 A-F

Granos grandes, suboblatos. Medidas: P $=62(65) 68 \mu \mathrm{m} ; \mathrm{E}=68$ (74) $80 \mu \mathrm{m} . \quad \mathrm{P} / \mathrm{E}=$ 0,87 .

Zonocolp(oid)orados. Número de aperturas 5. Ora circulares de $8 \mu \mathrm{m}$ de diámetro y engrosamiento de $2 \mu \mathrm{m}$; los colpoides miden $14 \mu \mathrm{m}$ de largo y se visualizan dificultosamente con el MO. La exina tiene un espesor de $5 \mu \mathrm{m}$ bajo las espinas, sexina $4 \mu \mathrm{m}$ y nexina $1 \mu \mathrm{m}$; en la zona interespinal la exina mide $3 \mu \mathrm{m}$, sexina 2 $\mu \mathrm{m}$ y nexina $1 \mu \mathrm{m}$. La ornamentación está constituida por espinas de $5 \mu \mathrm{m}$ de altura con ápices agudos y una distancia aproximada entre ellas de $10 \mu \mathrm{m}$, alternando con elementos positivos regularmente dispuestos.
MEB: Con altos aumentos $(6.500 \mathrm{x})$ pueden observarse los mamelones de las espinas con mayor claridad, los que cuentan en su parte basai con un anillo de nanoverrugas. La superficie ubicada entre las espinas está cubierta por nanoverugas y perforaciones laxamente dispuestas.

Distribución: Argentina (Corrientes, Entre Ríos, Santa Fe, Córdoba, La Pampa, Santiago del Estero y Buenos Aires).

\section{Modiolastrum lateritium (Hook.) Krapov.}

Figs. 1 D y 5 A-F

Granos grandes, oblato esferoidales. Medidas: $\mathrm{P}=63(76) 82 \mu \mathrm{m} ; \mathrm{E}=75(82,5) 88 \mu \mathrm{m}$. $\mathrm{P} / \mathrm{E}=0,92$.

Pentacolp(oid)orados, aperturas dispuestas en disposición espiraloide. Ora circulares de 5 $\mu \mathrm{m}$ de diámetro y engrosamiento de $3 \mu \mathrm{m}$; los colpoides miden $11 \mu \mathrm{m}$ de largo y se visualizan dificultosamente con el MO. La exina tiene un espesor de $3 \mu \mathrm{m}$ bajo las espinas, sexina $2 \mu \mathrm{m}$ y nexina $1 \mu \mathrm{m}$; en la zona interespinal la exina mide $2 \mu \mathrm{m}$, sexina $1 \mu \mathrm{m}$ y nexina $1 \mu \mathrm{m}$. La ornamentación está constituida por espinas de $5 \mu \mathrm{m}$ de altura con ápices agudos y una distancia aproximada entre ellas de 8-10 $\mu \mathrm{m}$, alternando con elementos positivos y negativos.

MEB: Permite observar con altos aumentos $(6.500 \mathrm{x})$ nanoverrugas y perforaciones del téctum ubicadas entre las espinas. Las nanoverrugas dispuestas laxamente, mostrando una tendencia a concentrarse alrededor de la base de los mamelones de las espinas.

Distribución: Argentina (Corrientes, Entre Ríos, Misiones, Buenos Aires).

\section{Modiolastrum malvifolium (Griseb.) K. Schum.}

Figs. 1 E y 6 A-F

Granos medianos, suboblatos. Medidas: P - 35 (37) $40 \mu \mathrm{m} ; \mathrm{E}=40(42) 44 \mu \mathrm{m} . \mathrm{P} / \mathrm{E}=0,88$. 

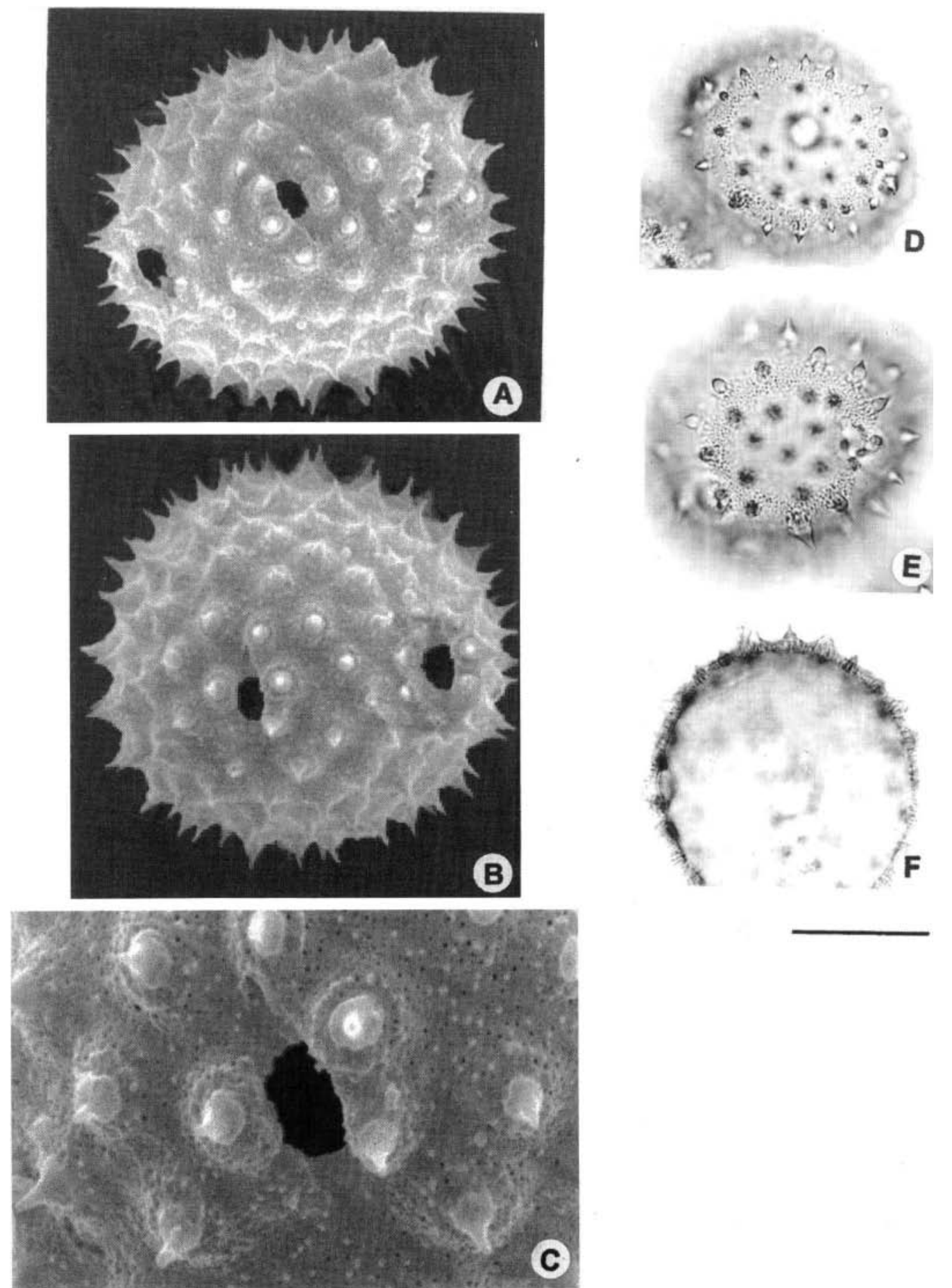

Fig. 4. Modiolastrum gilliesii. MEB: A y B: vista ecuatorial. C: detalle de escultura y apertura (Troncoso y Bacigalupo 2931). MO: D y E: vista ecuatorial, foco superior. F: corte óptico (Krapovickas y Cristóbal 22728). La escala equivale a $25 \mu \mathrm{m}$ en A y B, a $7 \mu \mathrm{m}$ en $\mathrm{C}$, a $35 \mu \mathrm{m}$ en D, E y F. 

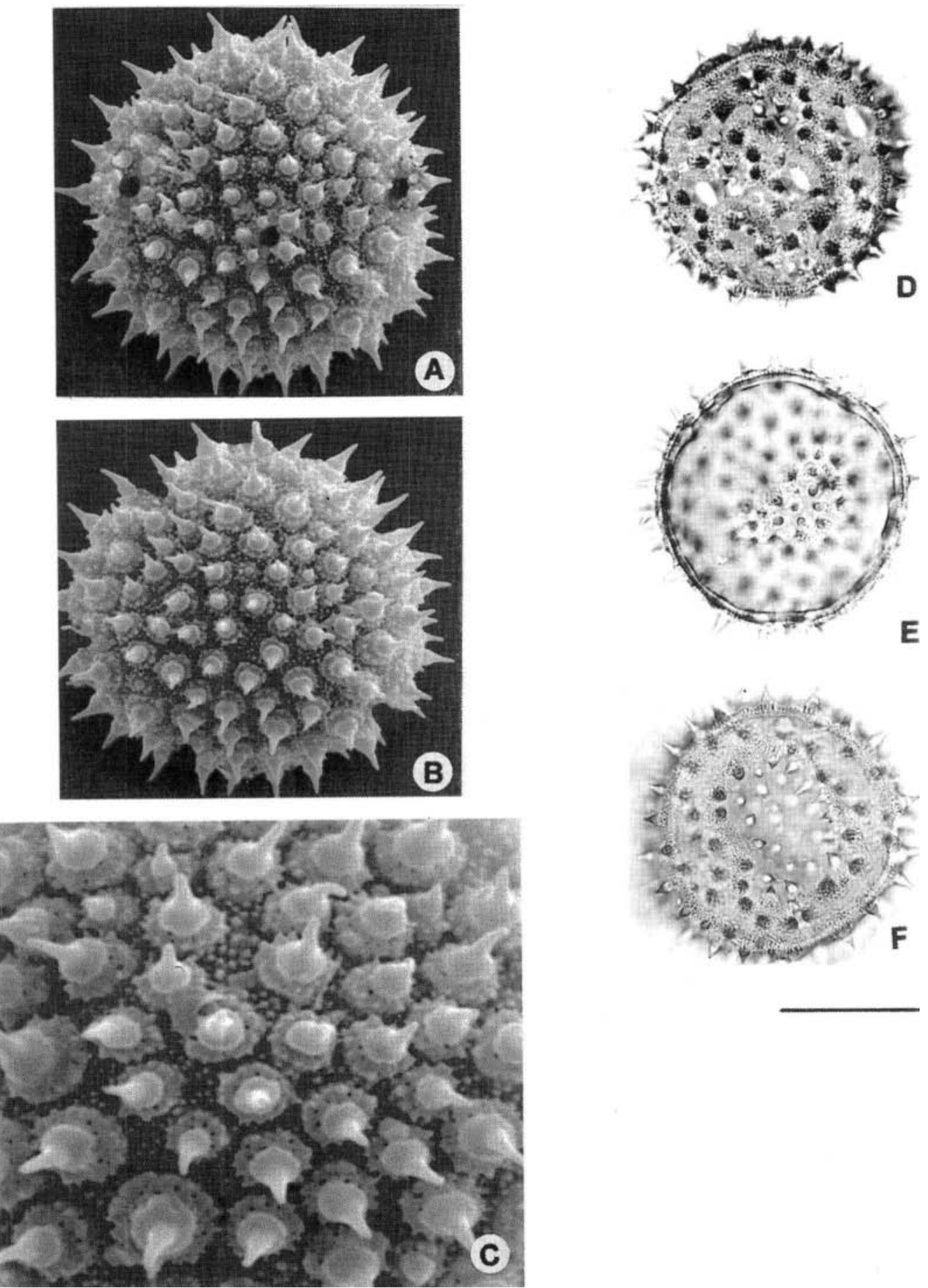

Fig. 3. Modiolastrum australe. MEB: A: vista ecuatorial. B: vista polar. C: detalle de escultura (Villamil 5628). MO: D: vista ecuatorial. E: vista polar. F: vista superficial (Villamil 5160). La escala equivale a $25 \mathrm{~mm}$ en A y B; a $15 \mathrm{~mm}$ en $\mathrm{C}$; a $40 \mathrm{~mm}$ en D, E y F. 

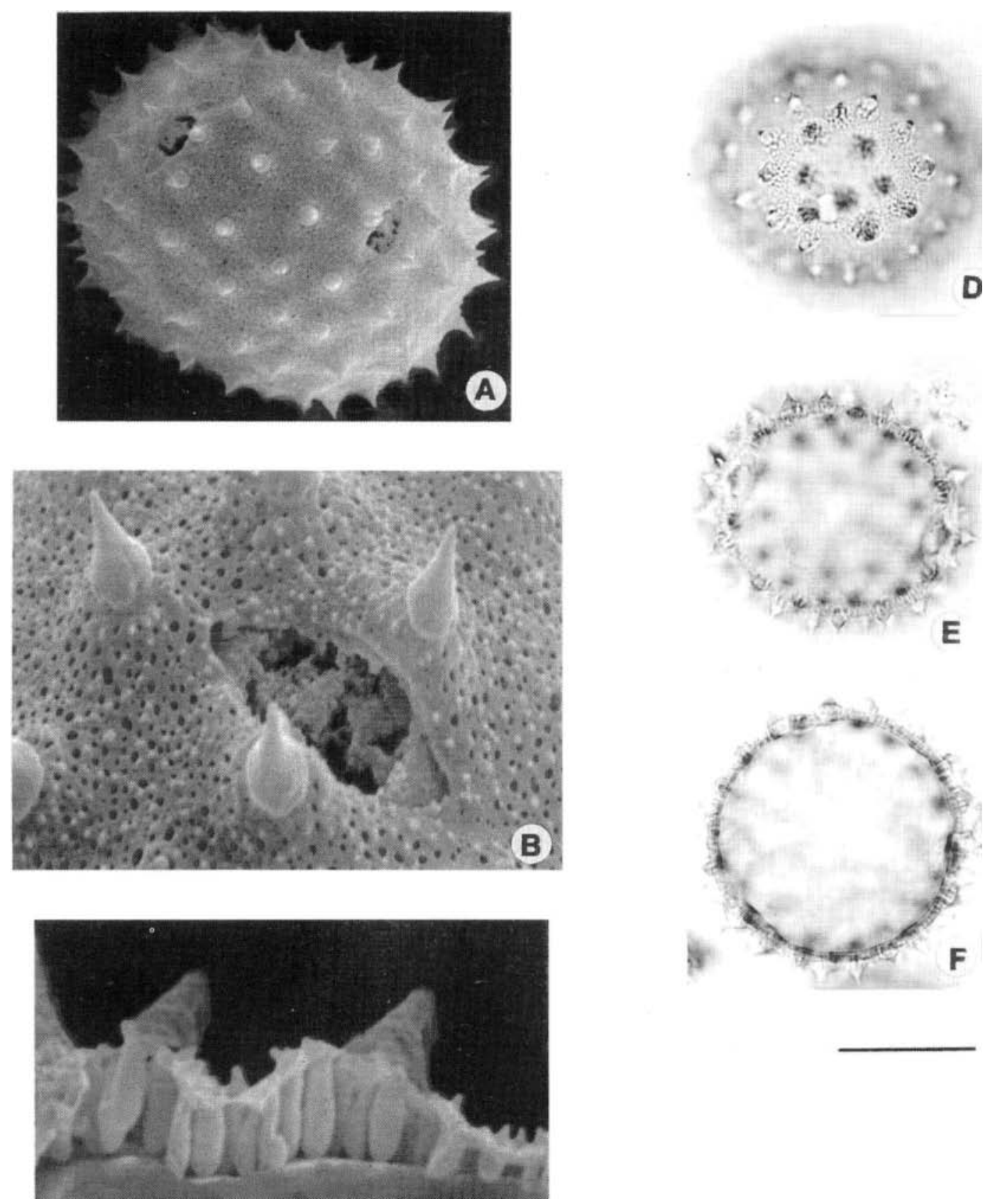

Fig. 6. Modiolastrum malvifolium. MEB: A: vista ecuatorial. B: detalle de escultura y apertura. C: corte de pared, detalle de escultura (Schinini et al. 11667). MO: D: vista ecuatorial, foco superior. E: vista ecuatorial, corte óptico. F: vista polar, corte óptico (Krapovickas y Cristóbal 22 728). La escala equivale a $15 \mu \mathrm{m}$ en A, a $5 \mu \mathrm{m}$ en B, a 2,6 $\mu \mathrm{m}$ en C y a $23 \mu \mathrm{m}$ en D, E y F. 

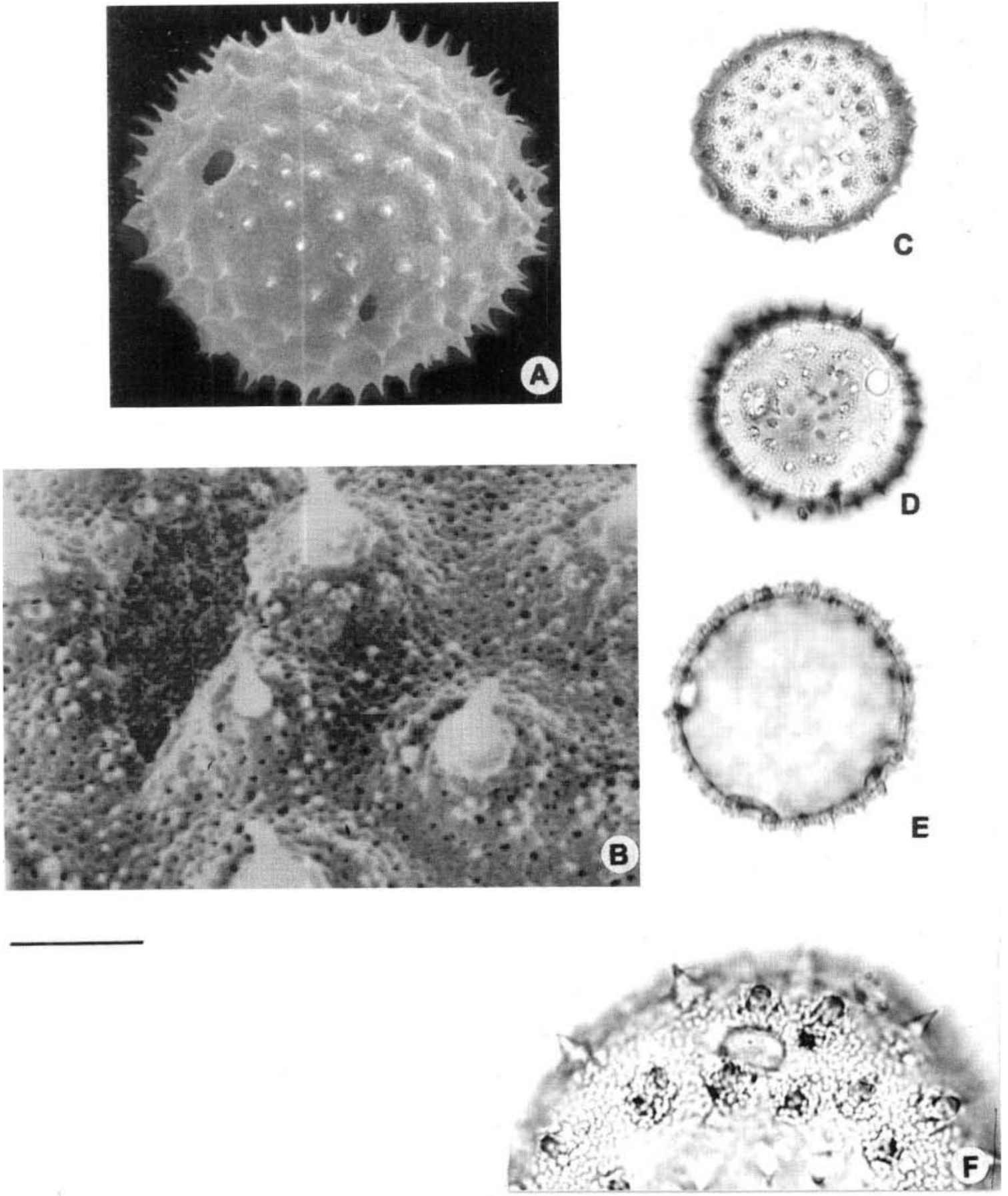

Fig. 5. Modiolastrum lateritium. MEB: A: vista ecuatorial. B: detalle de escultura y apertura (O. Ahumada y Schinini 4087). MO: C: vista ecuatorial foco superior. D: vista ecuatorial foco inferior. E: vista polar, corte óptico (Pedersen 12943). F: detalle de la escultura y apertura. La escala equivale a $27 \mu \mathrm{m}$ en A, a $5 \mu \mathrm{m}$ en B, a $50 \mu \mathrm{m}$ en C, D y E y a 22 $\mu \mathrm{m}$ en $\mathrm{F}$. 
Trizonocolporados. Ora circulares de $5 \mu \mathrm{m}$ de diámetro y engrosamiento de $2 \mu \mathrm{m}$; los colpoides miden $12 \mu \mathrm{m}$ de largo y se visualizan con dificultad con el MO. La exina tiene un espesor de $3 \mu \mathrm{m}$ bajo las espinas, sexina $2 \mu \mathrm{m}$ y nexina lum; en la zona interespinal la exina mide $1,5 \mu \mathrm{m}$, sexina $1 \mu \mathrm{m} y$ nexina $0,5 \mu \mathrm{m}$. La ornamentación está constituida por espinas de $4 \mu \mathrm{m}$ de altura con ápices agudos y una distancia aproximada entre ellas de 8-10 $\mu \mathrm{m}$, alternando con elementos positivos y negativos.

MEB : Se observan opérculos en los colpoides. Permite apreciar con altos aumentos $(5.000 \mathrm{x})$ espínulas y perforaciones del téctum ubicadas entre las espinas. También, en un grano roto, se puede observar en detalle la estructura de la exina y espínulas alternando con las espinas.

Distribución: Argentina (Corrientes, Chaco, Foimosa, Entre Ríos, Misiones, Santa Fe, Buenos Aires, Catamarca, Córdoba, Jujuy, La Rioja, Salta, Tucumán, San Luis y Santiago el Estero).

\section{Modiolastrum palustre (Ekman) Krapov.}

Figs. 1 F y Fig. 7 A-G

Granos medianos, suboblatos Medidas: $\mathrm{P}=$ 37 (39) $42 \mu \mathrm{m} ; \mathrm{E}=40$ (42) $44 \mu \mathrm{m}$. P/E - 0,88.

Trizonocolporados. Ora circulares de $6 \mu \mathrm{m}$ de diámetro y engrosamiento de $2 \mu \mathrm{m}$; los colpoides miden $10 \mu \mathrm{m}$ de largo y se visualizan con dificultad con el MO. La exina tiene un espesor de $4 \mu \mathrm{m}$ bajo las espinas, sexina 3 $\mu \mathrm{m}$ y nexina $1 \mu \mathrm{m}$; en la zona interespinal la exina mide $2 \mu \mathrm{m}$, sexina $1 \mu \mathrm{m}$ y nexina $1 \mu \mathrm{m}$; a nivel de los poros la nexina alcanza un espesor de $2 \mu \mathrm{m}$. La ornamentación está constituida por espinas de $4 \mu \mathrm{m}$ de altura y una distancia aproximada entre ellas de $10 \mu \mathrm{m}$, alternando con ellas, elementos positivos y perforaciones muy pequeñas.
MEB: Se observan los colporos con mayor claridad. Las espinas alternan con nanoverrugas dispuestas irregularmente, pero manteniendo una disposición anular en la base de los mamelones de las espinas; las perforaciones son redondeadas (fóveas) de diferente tamaño, pero menores de 1 u.m.

Distribución: Brasil (Sudeste del Estado de Paraná,)

\section{Tropidococcus pinnatipartitus (A. St.-Hil. \& Naudin) Krapov.}

Figs. 1 G y 8 A-G

Granos esferoidales grandes de 55 (59) 62,5 $\mu \mathrm{m}$ de diámetro. Dizonocolp(oid)orados, ora circulares de $6 \mu \mathrm{m}$ de diámetro, con engrosamiento anular endexínico de $2 \mu \mathrm{m}$. Los colpos de 12,5 $\mu \mathrm{m}$ de largo se visualizan con mucha dificultad con el MO, pero con más claridad en aquellos que han perdido las espinas.

El número de aperturas es de 12, distribuidas en una serie de 6 , a ambos lados del ecuador distancia entre las mismos entre 16 y $17 \mu \mathrm{m}$. La exina en la zona subespinal mide $6 \mu \mathrm{m}$ de espesor, la sexina $4 \mathrm{~m}$ y la nexina $2 \mu \mathrm{m}$; en la zona interespinal la exina tiene un espesor menor, de aproximadamente $4 \mu \mathrm{m}$, la sexina $2 \mu \mathrm{m}$ y la nexina $2 \mu \mathrm{m}$. La ornamentación está constituida por espinas de $5 \mu \mathrm{m}$ de altura, con una distancia aproximada entre ellas de $6 \mu \mathrm{m}$, alternando con elementos positivos y negativos.

MEB: Se observan los colporos, muy breves pasando poco el borde de los ora. Con altos aumentos $(4500$ x y 9000 x) puede apreciarse que las espinas alternan con nanoverrugas y perforaciones del téctum menores de $1 \mu \mathrm{m}$ de diámetro, redondeadas (fóveas) y elongadas (fósulas), dispuestas irregularmente.

Distribución: Brasil (Sudeste de Rio Grande do Sul). 

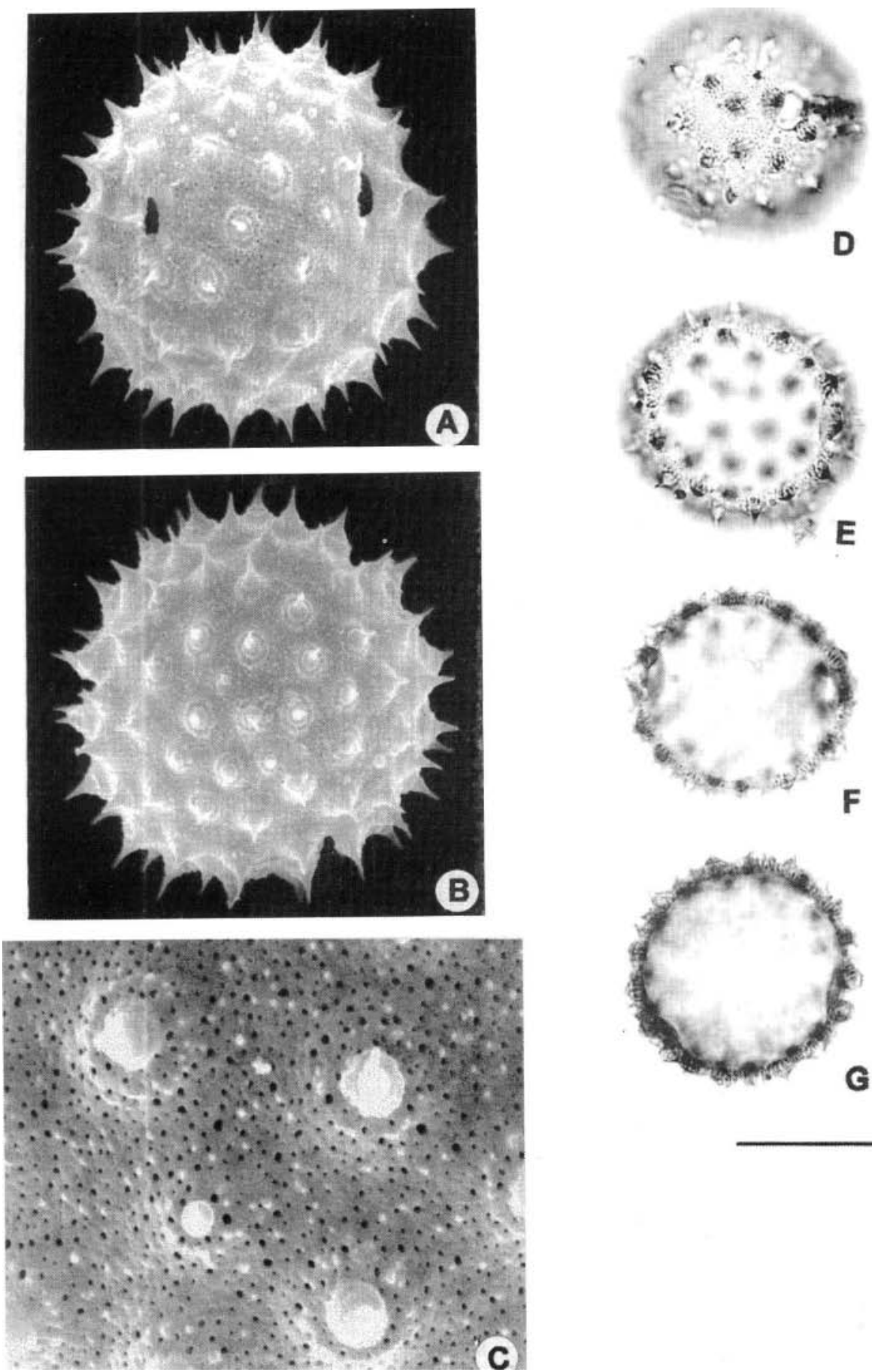

G

Fig. 7. Modiolastrum palustre. MEB: A: vista ecuatorial. B: vista polar. C: detalle de escultura (Hatschbach 15231). MO: D: vista ecuatorial, foco superior. E: vista ecuatorial, foco medio. F: vista ecuatorial, corte óptico. G: vista polar, corte óptico (Hastchbach 32784). La escala equivale a $18 \mu \mathrm{m}$ en A y B, a 4,5 $\mu \mathrm{m}$ en C y a $30 \mu \mathrm{m}$ en D, E ,F y G. 

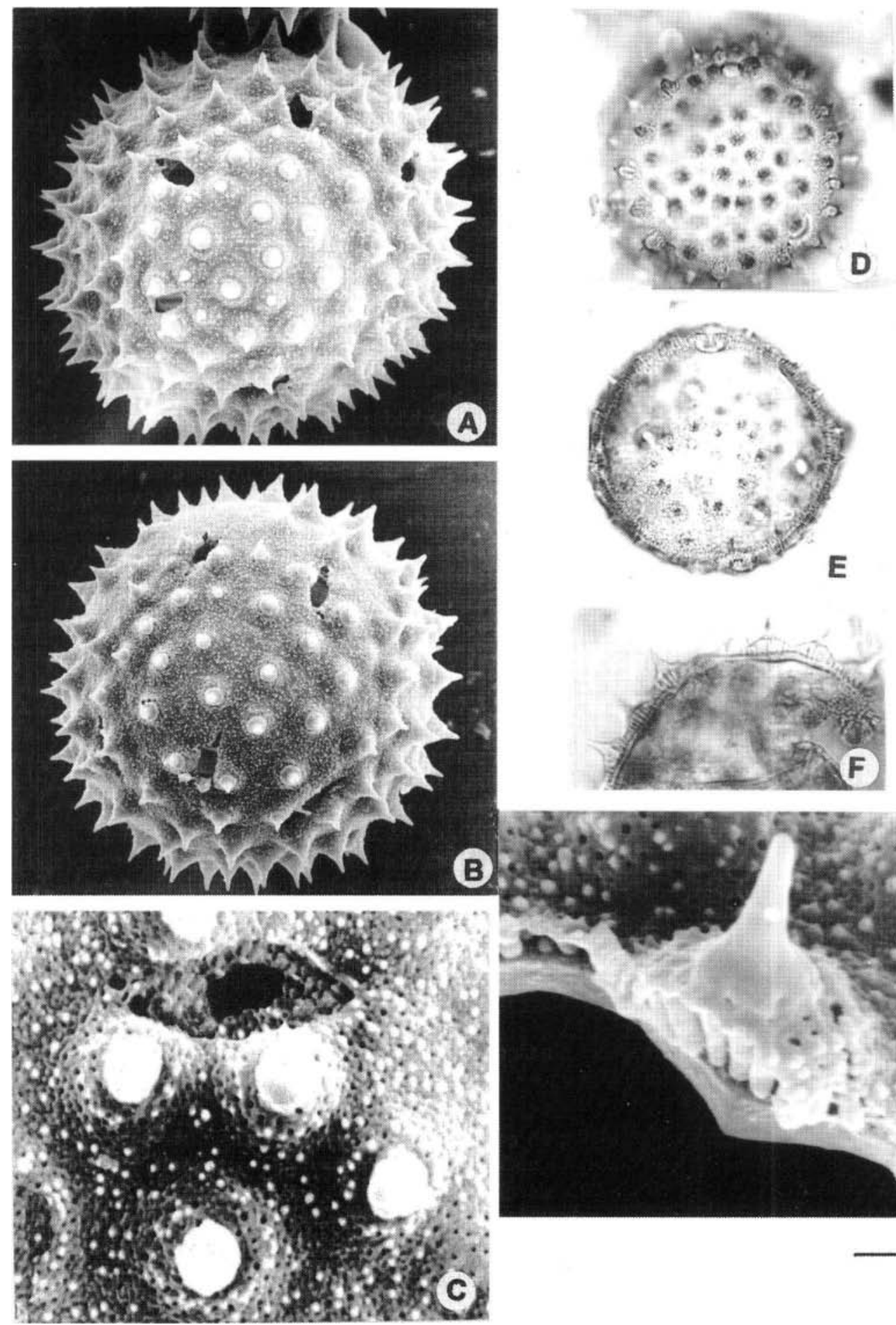

B
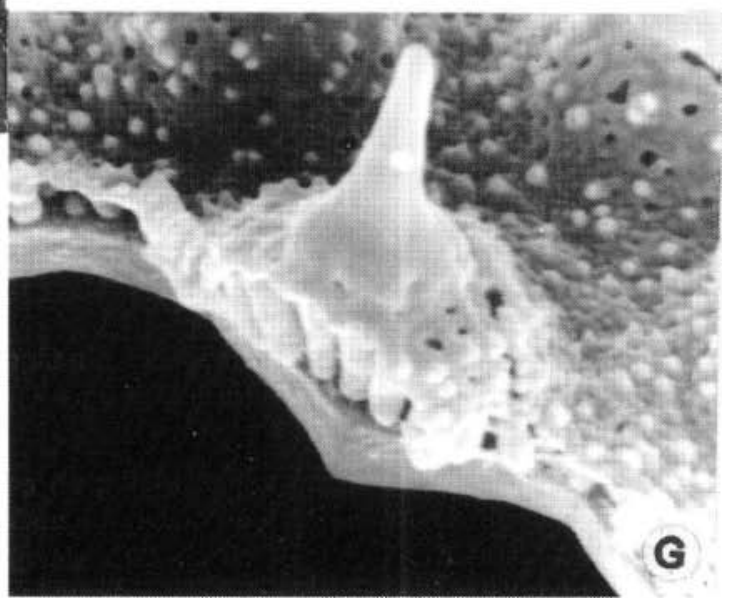

Fig. 8. Tropidococcus pinnatipartitus. MEB: A: vista polar. B: vista ecuatorial. C: detalle de ornamentación y colporo. G: corte de pared, mostrando su estructura y parte de la escultura. (Pedersen 114729). MO: D: vista polar, foco superior. E: vista ecuatorial. F: corte óptico (Krapovickasy Cristóbal 34209). La escala equivale a $20 \mu \mathrm{m}$ en A y $\mathrm{B}$, a 8 en $\mathrm{C}$, a $7 \mu \mathrm{m}$ en $\mathrm{G}$, a $34 \mu \mathrm{m}$ en D y E y $26 \mu \mathrm{m}$ en F. 
BONPLANDIA 12(1-4). 2003

\section{Cuadro comparativo de los principales caracteres de las especies estudiadas}

\begin{tabular}{|c|c|c|c|c|c|c|c|}
\hline & Tamaño & $\mu \mathrm{m}$ & Forma & Aperturas & $\begin{array}{l}\text { Altura } \\
\text { espinas } \\
\mu \mathrm{m}\end{array}$ & $\begin{array}{c}\text { Exina } \\
\text { s In }\end{array}$ & $\begin{array}{l}\text { Subespinal } \\
\text { terespinal } \\
\mu \mathrm{m}\end{array}$ \\
\hline $\begin{array}{l}\text { Modiola } \\
\text { caroliniana }\end{array}$ & $\begin{array}{l}\text { Medianos } \\
44,3\end{array}$ & s $43,3 \times$ & Oblato esferoidales & $\begin{array}{l}\text { Hexacolp(oid)orados, en } \\
\text { disposición espiraloide }\end{array}$ & 3 & 3 & 2 \\
\hline $\begin{array}{l}\text { Modiolastrum } \\
\text { malvifolium }\end{array}$ & Medianos & s $37 \times 43$ & Suboblatos & Trizonocolporados & 4 & 3 & 1,5 \\
\hline $\begin{array}{l}\text { Modiolastrum } \\
\text { palustre }\end{array}$ & Medianos & s $39 \times 42$ & Suboblatos & Trizonocolporados & 4 & 4 & 2 \\
\hline $\begin{array}{l}\text { Modiolastrum } \\
\text { gilliesii }\end{array}$ & Grandes & $65 \times 74$ & Suboblatos & Pentazonocolp(oid)orados & 5 & 5 & 3 \\
\hline $\begin{array}{l}\text { Modiolastrum } \\
\text { australe }\end{array}$ & Grandes & $63 \times 70$ & Oblato esferoidales & Pentazonocolp(oid)orados & 6 & 4 & 3 \\
\hline $\begin{array}{l}\text { Modiolastrum } \\
\text { lateritium }\end{array}$ & Grandes & $76 \times 82,5$ & Oblato esferoidales & $\begin{array}{l}\text { Pentacolp(oid)orados en } \\
\text { disposición espiraloide }\end{array}$ & 5 & 3 & 2 \\
\hline $\begin{array}{l}\text { Tropidococcus } \\
\text { pinnatipartitus }\end{array}$ & Grandes & $55(59) 62$ & Esferoidales & $\begin{array}{l}\text { Dizonocolp(oid)orados } 12 \\
\text { aperturas, } 6 \text { en cada hemisferio }\end{array}$ & 5 & 6 & 4 \\
\hline
\end{tabular}

\section{Clave para diferenciar especies}

1. Aperturas de 3 a 6, ubicadas en la zona ecuatorial. Granos suboblatos a oblato esferoidales. Exina subespinal 3 a $5 \mu \mathrm{m}$ e interespinal 1,5 a $3 \mu \mathrm{m}$ de espesor.

2. Granos medianos. Espinas 3-4 $\mu \mathrm{m}$ de altura.

3. Granos hexacolp(oid)orados, aperturas dispuestas en disposición espiraloide, oblato esferoidales. Exina subespinal $3 \mu \mathrm{m}$ e interespinal $2 \mu \mathrm{m}$ de espesor.

Modiola caroliniana

3. Granos trizonocolporados, suboblatos.

4. Exina subespinal $3 \mu \mathrm{m}$ e interespinal $1,5 \mu \mathrm{m}$ de espesor.

Modiolastrum malvifolium

4. Exina subespinal $4 \mu \mathrm{m}$ e interespinal $2 \mu \mathrm{m}$ de espesor.

Modiolastrum palustre

2. Granos grandes. Espinas 5-6 $\mu \mathrm{m}$ de altura.

5. Granos suboblatos. Pentazonocolp(oid)orados.

Modiolastrum gilliesii

5. Granos oblato esferoidales. 
6. Aperturas pentazonocolp(oid)oradas. Espinas densamente dispuestas, distancia entre ellas 5 $\mu \mathrm{m}$. Téctum con perforaciones sólo en la base de las espinas.

Modiolastrum australe

6. Aperturas pentacolp(oid)oradas en disposición espiraloide. Espinas laxamente dispuestas, distancia entre ellas 8-10 $\mu \mathrm{m}$. Téctum con perforaciones en toda la superficie del grano.

Modiolastrum lateritium

1. Aperturas 12, ubicadas en una serie a ambos lados del ecuador. Granos esferoidales. Exina subespinal, 6 u,m e interespinal, $4 \mu \mathrm{m}$ de espesor.

Tropidococcus pinnatipartitus

\section{Discusión y Conclusiones}

El polen de las especies de los géneros Modiola, Modiolastrum y Tropidococcus tiene en común el carácter más conspicuo, que distingue a la familia Malvaceae: la típica estructura de la exina, donde la sexina presenta en la base de las espinas los báculos más largos que en el resto del grano. Esto determina que la exina en corte óptico presente un aspecto ondulado (Figs. $6 \mathrm{C}$ y $8 \mathrm{G}$ ).

Los géneros Modiola y Modiolastrum tienen mucha afinidad palinológica. No obstante la combinación de los siguientes caracteres morfológicos: tamaño mediano; forma oblato esferoidal y aberturas ecuatoriales dispuestas en disposición espiraloide, sólo se presenta en Modiola caroliniana, cuyo número cromosómico básico es $\mathrm{x}=9$.

Se ha hallado coincidencia en la mayoría de los caracteres descriptos por Christensen (1986) en el polen de Modiola caroliniana, sólo difieren ligeramente el tamaño de los granos y significativamente el espesor de la exina, $7 \mu \mathrm{m}$ en la descripción de Christensen y $4 \mu \mathrm{m}$ (considerando la zona de mayor espesor) en este trabajo.

Heusser (1971) cita Modiola caroliniana para Santiago de Chile y brinda datos del polen, observado con microscopio óptico. Cotejando estos datos con los obtenidos, el autor se refiere a la exina como de $2 \mu \mathrm{m}$ de espesor $\sin$ especificar a qué nivel se realizaron las mediciones, lo cual es impreciso ya que si bien esta medida es coincidente en la zona interespinal, en la zona subespinal la exina de esta especie tiene $4 \mu \mathrm{m}$ de espesor. Hashmi (1970) estudia el polen de Modiola caroliniana procedente de Texas, EEUU. La medida promedio de los granos de polen de esta especie que el autor menciona es de $59 \mu \mathrm{m}$, con un rango de 47-75 $\mu \mathrm{m}$, lo cual significa un tamaño mayor al de la misma especie de Argentina. Referente al espesor de la exina, no aclara si se trata de la zona subespinal o interespinal. Los dos últimos autores se refieren a la ubicación de las aperturas como estefanocolporadas y zonicolporadas respectivamente, mientras que según las observaciones realizadas en este trabajo su disposición es espiraloide.

Modiolastrum gilliesii y $M$. malvifolium son descriptos por Christensen (1986) como rugulados y rodeados por pocas nanoverrugas. Las mismas especies analizadas en este trabajo presentan los granos de polen equinados y, en el caso de $\boldsymbol{M}$. malvifolia, las espinas alternan con espínulas y perforaciones. En cuanto a las aberturas se coincide en el número y posición que brinda la autora ( 3-6) ubicadas usualmente en posición zonal, pero algunas veces en disposición espiraloide.

En el género Modiolastrum $(\mathrm{x}=5) \boldsymbol{M}$. malvifolium y $\boldsymbol{M}$. palustre comparten en el polen los caracteres considerados más primitivos dentro de la familia (Christensen 1986): tricolporados, medianos, suboblatos, granulaciones escasas, espinas cortas $(3-4 \mu \mathrm{m}$ de altura). Por lo tanto, los caracteres palinológicos relacionan estas especies a pesar de que se trata de plantas muy diferente por su exomorfología.

Modiolastrum australe, $M$. gilliesii $y$ M. lateritium son muy afines teniendo en cuenta caracteres detectables con MO como, forma, tamaño, número y disposición de aperturas, 
altura de las espinas y espesor de la exina. Las observaciones y fotografías con el MEB permiten determinar diferencias considerables: espinas de base ancha, muy densamente dispuestas en $M$. australe que dificultan la observación de las aberturas y el mismo tipo de espinas pero más distanciadas en $M$. gilliesü. El téctum presenta perforaciones sólo en la base de las espinas en $M$. australe y en toda la superficie del grano en $M$. gilliesü y $M$. lateritium.

La segregación realizada por Krapovickas (2003) de M. pinnatipartitus a Tropidococcus, con un número básico de cromosomas $\mathrm{x}=12$, es sustentada por las observaciones palinológicas realizadas en esta especie, dado que el polen de $T$. pinnatipartitus es claramente distinto de las especies de Modiolastrum. La diferencia fundamental radica en el número y la posición de las aperturas, las cuales están dispuestas en una serie de 6 aperturas ubicadas a ambos lados del ecuador (dizonoaperturados), y el mayor espesor de la exina, tanto a nivel infraespinal como interespinal. Cabe puntualizar que es la única especie que presenta granos de polen esferoidales, si bien la forma del grano es un factor de menor importancia en términos filogenéticos.

Teniendo en cuenta el número básico de cromosomas de cada género, se apoya la opinión de Erdtman (1963) y Christensen (1986) de que a mayor número de aperturas, mayor grado de evolución. Esto se fundamenta en que, si bien los tres géneros tienen número básico de cromosomas bajo, hay una pequeña diferencia y ésta se relaciona con el número de aperturas de los granos de polen: Modiolastrum $\mathrm{x}=5$, aberturas 3 a 5 ; Modiola $\mathrm{x}=9$, aberturas 6; Tropidococcus $\mathrm{x}=\mathrm{T} 2$, aberturas 12 .

Las especies estudiadas pueden dividirse en cinco tipos polínicos, teniendo en cuenta el número y disposición de las aberturas.

Tipo trizonocolporado: Modiolastrum malvifolium y $M$. palustre.

Tipo pentazonocolp(oid)orado: Modiolastrum australe, M. gilliesü.

Tipo pentacolp(oid)orado, con aperturas en disposición espiraloide: Modiolastrum lateritium.
Tipo hexacolp(oid)orado, con aperturas en disposición espiraloide: Modiola caroliniana.

Tipo dizonocolp(oid)orado, dispuestos en una serie de seis aperturas a ambos lados del ecuador: Tropidococcus pinnatipartitus.

\section{Agradecimientos}

Deseo expresar mi agradecimiento a las autoridades del Instituto de Botánica del Nordeste (IBONE), quienes permitieron mi acceso al herbario y a la biblioteca. En especial al Ing. Antonio Krapovickas, quien me ha facilitado el material para la realización de este trabajo, como también por su asesoramiento, consejos y lectura crítica del manuscrito, como especialista en la familia.

\section{Bibliografía}

CHRISTENSEN, P.B. 1986. Pollen morphological studies in the Malvaceae. Grana 25(2):95-1 17.

ERDTMAN, G. 1960. The acetolysis method. Svensk.Bot. Tidskr. 54(4):561-564.

. 1963. Palynology. Advanc. Bot. Res. I: 149. 208

1966. Pollen Morphology and Plant Taxonomy and Plant. Angiosperms. Hafner Publ. Co., New York.

HASHMI, S.H. 1970. The Palynology of the Malvaceae of Texas. Submitted to the Graduate College of Texas A \& M University in Partial fulfillment of the requirement for the degree of Doctor of Philosophy. (No publicado).

HEUSSER, C.J. 1971. Pollen and Spores of Chile Modern Types of Pteridophyta, Gymnospermae and Angiospermae. The University of Arizona Press. Tucson.

KRAPOVICKAS, A. 2003. Tropidococcus Krapov., nuevo genero de Malvaceas. Bonplandia 12(1-4): 63-66.

PUNT, W., S. BLACKMORE, S. NILSSON \& A. LE THOMAS. 1994. Glossary of Pollen and Spores. Terminology. LPP Foundation, LPP Contributions Serie № 1. University of Utrecht, The Netherlands.

Original recibido el 23 de diciembre de 2002; aceptado el 30 de abril de 2003. 